\title{
Three-dimensional free vibration analysis of thick laminated circular plates
}

\author{
Sumit Khare $^{1 *}$, N. D. Mittal ${ }^{2}$ \\ ${ }^{1,2}$ Department of Mechanical Engineering, Maulana Azad National Institute of Technology, Bhopal-462003, INDIA \\ "Corresponding Author: e-mail: sumitkhare8686@ gmail.com, Tel +91-8109152818
}

\begin{abstract}
In this communication, a numerical analysis regarding free vibration of thick laminated circular plates, having free, clamped as well as simply-supported boundary conditions at outer edges of plates is presented. The employment of finite element is made in this communication. The finite element methodology operates on the basis of three-dimensional theory of elasticity and was employed to assess the natural frequencies for laminated circular plates of various thickness-to-outer radius ratios. The first five natural modes of flexural vibrations for different boundary conditions are presented in pictorial forms. Verification of the accuracy of the results was made using the necessary convergence analysis and checked using literature results.
\end{abstract}

Keywords: Laminated Composite Circular Plate; Natural Frequency; Mode Shape; Finite Element Method.

DOI: http://dx.doi.org/10.4314/ijest.v8i2.2

\section{Introduction}

Laminated circular plates are commonly used structural component having a broad application in aerospace, civil, mechanical, nuclear, electronic as well as marine engineering. An essential prerequisite in designing and performance assessment of mechanical systems is understanding the free vibration behavior of different plate components. The dynamic response of complex engineering systems is intimately linked with plate response frequencies as well as vibration mode shapes. A thorough analysis of free vibration data is often useful in arriving at the resonant behavior and fatigue stress estimation at vulnerable machine locations.

A comprehensive survey of early investigation on free vibration studies of circular plates carried out by Leissa (1969) as well as Liew et al. (1995) noted that classical thin plate theory (CTPT) and Mindlin plate theory were majorly applied by investigators. It is understood that CTPT, which ignores the influences of transverse shear deformation as well as normal strain, overestimate the vibration frequencies and error increases with plate thickness. However, various shear deformation as well as higher-order theories which include the shear deformation and normal strain, have been proposed in the computations concerning thick plates during the past few decades. Rao and Prasad (1975) analysed the natural vibrations of annular plates considering the influences of shear deformation as well as rotatory inertia. Irie et al. (1982) presented the vibration characteristics of Mindlin annular plates in nine groupings of free, simply supported and clamp boundary condition of inner and outer edge conditions using Bessel function.

Also, Liew et al.(2000),Wu et al.(2002), Han and Liew (1999) and Wang (2004) discussed the free vibration of circular, annular and sector plates employing differential quadrature method based on the classical thin plate theory or Mindlin plate theory. Lin and Tseng (1998) studied the free vibration characteristics of polar orthotropic laminated circular and annular plates by using a finite element method having first order shear deformation theory. Hosseini-Hashemi et al. (2010) provided an precise closed-form frequency equation for thick circular plates using a third-order shear deformation theory. Hosseini-Hashemi et al. (2012) presented an precise closed-form solution for free flexural vibration of thick laminated circular plates with an attached rigid core using the first-order shear deformation theory. Senjanovic (2014) utilised the modified Mindlin theory to examine the free vibration of thick circular plates. Sharma (2014) investigated the free vibration problem involving averagely thick antisymmetric laminated annular sector plates having edge-supports that are elastic established on first order shear deformation theory using the differential quadrature method. Powmya and Narasimhan (2015) studied the free vibration behavior of polar orthotropic circular as well as annular plates by Chebyshev collocation technique based on first order shear deformation theory. 
The analysis of relatively thick plates is a challenging task. Most two - dimensional theories, if applied for the analysis of such thick plates, result in significant errors due to their inherent limitations. Such theories are almost always based on some simplifying assumptions and consequently offer a great deal of convenience in analysis. The expressions, thus obtained are simple in nature and easy to work with but are likely to be error prone in some cases. A three dimensional analysis, though more complicated, succeeds in getting rid of these errors to a considerable extent and goes far beyond a typical two - dimensional analysis in terms of utility, accuracy and extractable information. The three - dimensional research endeavors listed in literature are relatively few and the reasons are pretty understandable.

So and Leissa (1998), Zhou et al. (2003), Kang and Liessa (1998), Liew and Yang (1999,2000) and Kang (2003) presented the three-dimensional method of analysis for free vibration of circular as well as annular plates employing Ritz method. Liu and Lee (2000) employed the finite element method to investigate three-dimensional vibrations of thick circular and annular plates. Malekzadeh (2010) analysed the three-dimensional free vibration analysis of thick fuctionally graded annular plates influenced by the thermal environment.The three-dimensional analyses of circular and annular plates resting on Pasternak elastic foundation and Winkler foundation studied by Liew et al.(1996), Zhou et al. (2006), Hashemi et al. (2008). Houmat (2004) investigated the free vibration of annular sector plates employing finite element scheme. Komur et.al (2010) has conducted a buckling analysis for laminated composite plates having an elliptical/circular hole centered in the plate using finite element method (FEM) using ANSYS finite element software. Chen and Ren (1998) studied the transverse vibration of thin circular and annular plates with variable thickness using finite element method. Liang et al. (2007) used three-node annular finite elements to compute the natural frequencies of circular annular plates of polar orthotropy and non-uniform thickness. Ranjan and Ghosh (2009) studied the free and forced Transverse vibration behavior of a thin spinning disk having distributed patch attached to it as well as discrete point masses at the peripheryof plate using finite element analysis. Malekzadeh (2010) analysed a three-dimensional elasticity solution system, which addressed the free vibration analysis of thick-laminated circular and annular plates, which rested on two-parameter elastic foundation. In this paper the effects of different fiber orientation angle, thickness to radii ratio, with free, clamped and simply-supported boundary conditions of plates on the free vibration responses are discussed in detail using finite element scheme, with roots in three-dimensional theory of elasticity. Verification of accuracy as well as the numerical reliability was made using standard convergence principles while the study was compared with those existing in literature.

\section{The basic formulations}

Consider a thick, laminated annular plate having inner radius $R_{1}$, outer radius $R_{2}$, total thickness $h$ and fiber orientation angle $\beta$. An orthogonal cylindrical coordinates $(\mathrm{r}, \theta, \mathrm{z})$ are used as depicted in Figure 1, where $\mathrm{u}, \mathrm{v}$ and $\mathrm{w}$ denotes the displacement of any point of the plate in the $\mathrm{r}, \theta$ and $\mathrm{z}$ direction, respectively.

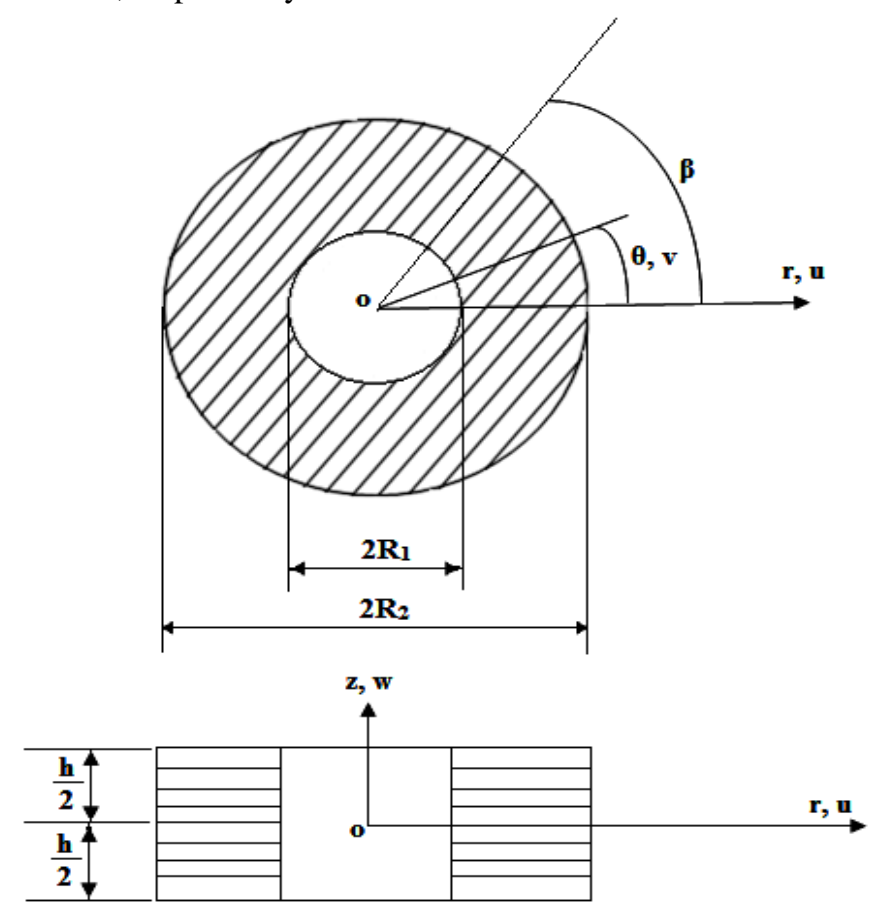

Figure 1 Geometry and coordinate system of the annular plate 
The displacement field that satisfy the three-dimensional elasticity equation of motion of circular plates, designated by $u, v$, and $w$ are assumed as

$u=U(r, z, t) \cos n \theta$

$v=V(r, z, t) \sin n \theta$

$w=W(r, z, t) \cos n \theta$,

where $n$ and $t$ represent the wave numbers (circumferential) as well as time, respectively. The displacements are afterwards used in the strain-displacement associations while the strains are consequently stated with respect to displacements. The stress-strain expressions sequentially direct to stresses that are as well stated with respect to displacements (Liu and Lee , 2000).

The constitutive relations representing that of arbitrary lamina for annular plate may be stated as,

$$
\left\{\begin{array}{l}
\sigma_{z} \\
\sigma_{\theta} \\
\sigma_{r} \\
\tau_{r \theta} \\
\tau_{r z} \\
\tau_{\theta z}
\end{array}\right\}=\left[\begin{array}{llllll}
C_{11} & C_{12} & C_{13} & 0 & 0 & C_{16} \\
C_{12} & C_{22} & C_{23} & 0 & 0 & C_{26} \\
C_{13} & C_{23} & C_{33} & 0 & 0 & C_{36} \\
0 & 0 & 0 & C_{44} & C_{45} & 0 \\
0 & 0 & 0 & C_{45} & C_{55} & 0 \\
C_{16} & C_{26} & C_{36} & 0 & 0 & C_{66}
\end{array}\right]\left\{\begin{array}{l}
\varepsilon_{z} \\
\varepsilon_{\theta} \\
\varepsilon_{r} \\
\varepsilon_{r \theta} \\
\varepsilon_{r z} \\
\varepsilon_{\theta z}
\end{array}\right\},
$$

where $[C]$ is the material stiffness matrix (Malekzadeh, 2010).

The Hamilton's principle is required for the modeling of vibrating plates using finite element (Liu and Lee, 2000):

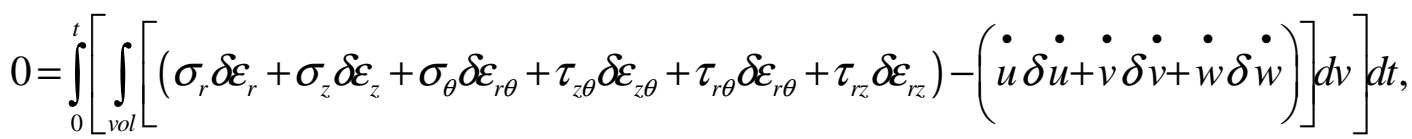

here, $u, v$ as well as $w$ represent velocity constituents along the three co-ordinates. Following the substitution of stresses as well as strains obtained through strain-displacement as well as stress-strain associations into above stated expression, we conclude with a variational kind in which the only main variables are the three displacements. The universal procedure of the finite element scheme then follows. If a single finite element becomes utilized in the integration, the element equation may be obtained as

$[m]\{\dot{U}\}+[k]\{U\}=0$

here, $\{U\}^{T}=\left[U_{1}, U_{2}, \ldots, U_{m}, V_{1}, V_{2}, \ldots, V_{m}, W_{1}, W_{2}, \ldots, W_{m}\right], \mathrm{m}$ refers to number of nodes in an element, [m] and $[\mathrm{k}]$ are the element mass and stiffness matrices.

The universal expression of motion may be obtained through the assembly of all the element expressions:

$$
[M]\{\ddot{X}\}+[K]\{X\}=0
$$

which corresponds to an eigenvalue equation of the following form:

$$
[K]\{x\}=\lambda[M]\{x\}=0
$$

The eigenvalue $\lambda$ represents the square of vibration frequency $\omega$ and eigenvector $\{x\}$ denotes the corresponding mode shape. 


\section{Material and methods}

A finite element analysis was made for obtaining the first five natural frequencies using three-dimensional 'SOLID185' of ANSYS. In addition, SOLID185 Structural Solid is appropriate for modeling common 3-D solid structures. As demonstrated in figure 2, the element contains eight nodes with three degrees of freedom at each node: translations in the nodal $\mathrm{x}$, $\mathrm{y}$, and $\mathrm{z}$ directions. (ANSYS Inc., 2009). The Block-Lanczos algorithm is utilised in free vibration analysis of thick laminated circular plates (Figure 3).
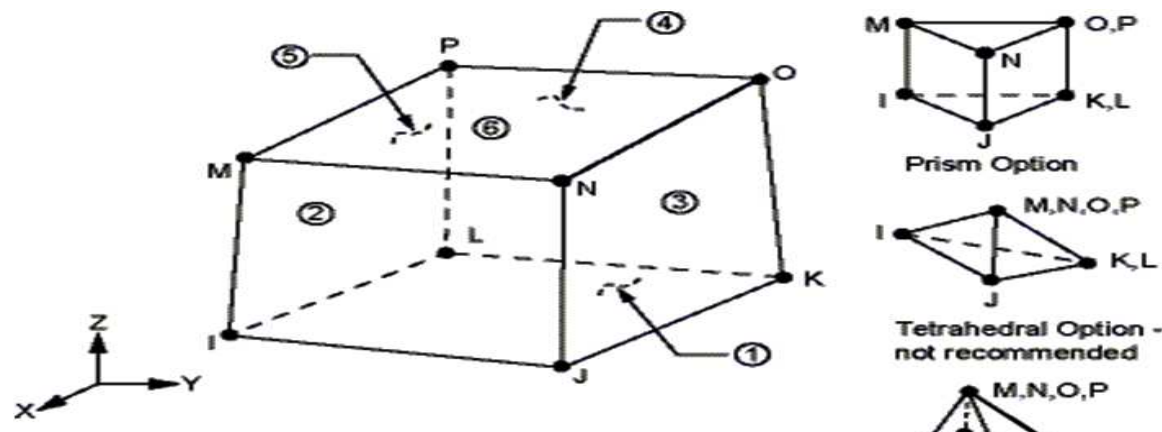

Tetrahedral Option
not recommended

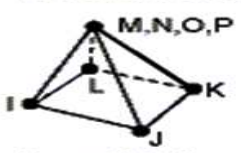

Pyramid Option -

not recommended

Figure 2 Eight noded SOLID185 element

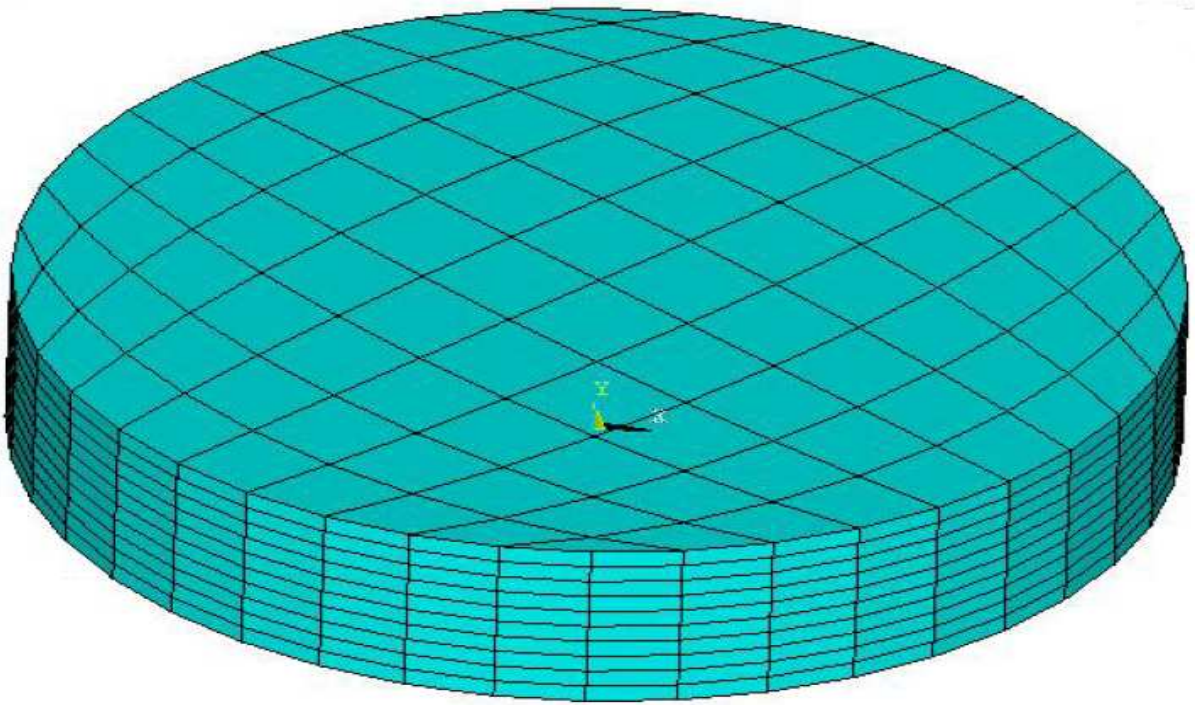

Figure 3 Finite element model of a thick laminated circular plate

Mechanical properties of glass/epoxy laminated plates are listed in Table 1.

Table 1. Mechanical properties of the glass/epoxy laminated plates (Reddy, 2004)

\begin{tabular}{llllll}
\hline $\mathrm{E}_{1}(\mathrm{GPa})$ & $\mathrm{E}_{2}(\mathrm{GPa})$ & $\mathrm{G}_{12}(\mathrm{GPa})$ & $\mathrm{G}_{13}(\mathrm{GPa})$ & $\mathrm{G}_{23}(\mathrm{GPa})$ & $\mathrm{V}$ \\
\hline 53.781 & 17.927 & 8.9635 & 8.9635 & 4.4475 & 0.25 \\
\hline
\end{tabular}




\section{Results and Discussion}

The current investigation was primarily validated via performing convergence study of non-dimensional frequency parameter $\Omega$ defined by $\Omega=\omega R_{2}^{2} \sqrt{\rho h / D}$, where $D=E h^{3} / 12\left(1-v^{2}\right)$, of isotropic circular plates ( $\left.\mathrm{v}=1 / 3\right)$ with respect to number of elemental divisions $(\mathrm{N})$ as well as through matching up of current results with literature reported cases. The number in parenthesis (n, s) indicates the number of nodal diameters $\mathrm{n}$, in the $\mathrm{s}^{\text {th }}$ mode of vibration. The rate of convergence of the first five frequency parameters for isotropic circular plates with clamped, simply supported and free boundary conditions are presented in Table 2-4, for thickness-radius ratios varying from 0.1 to 0.5 in step of 0.1 . It can be seen from that $\mathrm{N}=15$, is sufficient to obtain satisfactory convergence for first five frequency parameters.

Table 2. Convergence behavior of the first five frequency parameter $\Omega=\omega R_{2}^{2} \sqrt{\rho h / D}$ for isotropic circular plate with clamped boundary condition

\begin{tabular}{|c|c|c|c|c|c|}
\hline \multirow[t]{2}{*}{$\mathrm{N}$} & \multicolumn{5}{|c|}{ Mode number $(\mathrm{n}, \mathrm{s})$} \\
\hline & $1(0,0)$ & $2(1,0)$ & $3(2,0)$ & $4(0,1)$ & $5(3,0)$ \\
\hline \multicolumn{6}{|c|}{ (a) $\mathrm{h} / \mathrm{R}_{2}=0.1$} \\
\hline 7 & 10.4283 & 21.7720 & 35.5635 & 42.1115 & 51.8218 \\
\hline 9 & 10.4826 & 21.7897 & 35.6348 & 43.0988 & 51.6304 \\
\hline 11 & 10.2066 & 21.0863 & 34.0250 & 40.1456 & 48.7675 \\
\hline 13 & 10.0848 & 20.6712 & 33.2306 & 38.4154 & 47.5493 \\
\hline 15 & 10.0424 & 20.5753 & 33.0433 & 37.9726 & 47.3433 \\
\hline 17 & 10.0253 & 20.4796 & 33.0105 & 37.7931 & 47.2276 \\
\hline \multicolumn{6}{|c|}{ (b) $h / R_{2}=0.2$} \\
\hline 7 & 9.8278 & 18.8888 & 29.1105 & 33.6838 & 40.2617 \\
\hline 9 & 9.6245 & 18.8009 & 29.0413 & 34.0394 & 40.0299 \\
\hline 11 & 9.4536 & 18.4006 & 28.2071 & 32.4892 & 38.6473 \\
\hline 13 & 9.3676 & 18.1593 & 27.7543 & 31.5463 & 37.9937 \\
\hline 15 & 9.3421 & 18.1025 & 27.652 & 31.2997 & 37.8875 \\
\hline 17 & 9.3275 & 18.0371 & 27.6322 & 31.1883 & 37.8137 \\
\hline \multicolumn{6}{|c|}{ (c) $h / R_{2}=0.3$} \\
\hline 7 & 8.6985 & 16.0522 & 23.7183 & 27.0007 & 31.8281 \\
\hline 9 & 8.6739 & 15.9754 & 23.6418 & 27.1761 & 31.6365 \\
\hline 11 & 8.5511 & 15.7110 & 23.1393 & 26.2463 & 30.8234 \\
\hline 13 & 8.4871 & 15.5522 & 22.8576 & 25.6635 & 30.4271 \\
\hline 15 & 8.4673 & 15.5135 & 22.7934 & 25.5069 & 30.36 \\
\hline 17 & 8.4557 & 15.4683 & 22.7798 & 25.4341 & 30.3115 \\
\hline \multicolumn{6}{|c|}{ (d ) $\mathrm{h} / \mathrm{R}_{2}=0.4$} \\
\hline 7 & 7.7703 & 13.6761 & 19.6995 & 22.1995 & 26.0189 \\
\hline 9 & 7.7439 & 13.6095 & 19.6308 & 22.3029 & 25.8622 \\
\hline 11 & 7.6528 & 13.4285 & 19.2916 & 21.6759 & 25.0356 \\
\hline 13 & 7.6040 & 13.3172 & 19.0994 & 21.2753 & 25.0356 \\
\hline 15 & 7.5883 & 13.2889 & 19.0545 & 21.1650 & 24.9871 \\
\hline 17 & 7.5791 & 13.2566 & 19.0442 & 21.1132 & 24.9517 \\
\hline \multicolumn{6}{|c|}{ (e) $h / R_{2}=0.5$} \\
\hline 7 & 6.9352 & 11.7888 & 16.7370 & 18.7443 & 21.9125 \\
\hline 9 & 6.9094 & 11.7318 & 16.6768 & 18.3830 & 21.7812 \\
\hline 11 & 6.8397 & 11.5998 & 16.4258 & 18.3494 & 21.3643 \\
\hline 13 & 6.8016 & 11.5169 & 16.2832 & 18.0496 & 21.1580 \\
\hline 15 & 6.7887 & 11.4950 & 16.2492 & 17.9655 & 21.1196 \\
\hline 17 & 6.7812 & 11.4705 & 16.2406 & 17.9372 & 21.0914 \\
\hline
\end{tabular}


Table 3. Convergence behavior of the first five frequency parameter $\Omega=\omega R_{2}^{2} \sqrt{\rho h / D}$ for isotropic circular plate with simply

\begin{tabular}{|c|c|c|c|c|c|}
\hline \multirow[t]{2}{*}{$\mathrm{N}$} & \multicolumn{5}{|c|}{ Mode number $(\mathrm{n}, \mathrm{s})$} \\
\hline & $1(0,0)$ & $2(1,0)$ & $3(2,0)$ & $4(0,1)$ & $5(3,0)$ \\
\hline \multicolumn{6}{|c|}{ (a) $\mathrm{h} / \mathrm{R}_{2}=0.1$} \\
\hline 7 & 4.9780 & 14.0886 & 25.9652 & 31.1872 & 40.6181 \\
\hline 9 & 5.0141 & 14.1190 & 26.0418 & 31.8331 & 40.3566 \\
\hline 11 & 4.9346 & 13.8138 & 25.1410 & 30.0928 & 38.6387 \\
\hline 13 & 4.8952 & 13.6254 & 24.7280 & 29.1561 & 37.9233 \\
\hline 15 & 4.8794 & 13.5871 & 24.6232 & 28.9016 & 37.7744 \\
\hline 17 & 4.8758 & 13.5502 & 24.6017 & 28.8128 & 37.6903 \\
\hline \multicolumn{6}{|c|}{ (b) $h / R_{2}=0.2$} \\
\hline 7 & 4.8456 & 13.1048 & 23.0095 & 27.1966 & 34.2712 \\
\hline 9 & 4.8419 & 13.0538 & 22.9288 & 27.4563 & 33.9390 \\
\hline 11 & 4.7968 & 12.8488 & 22.3488 & 26.3701 & 32.8608 \\
\hline 13 & 4.7730 & 12.7277 & 22.0613 & 25.7400 & 32.3697 \\
\hline 15 & 4.7642 & 12.6999 & 21.9910 & 25.5725 & 32.2699 \\
\hline 17 & 4.7606 & 12.6699 & 21.9742 & 25.5015 & 32.2031 \\
\hline \multicolumn{6}{|c|}{ (c) $\mathrm{h} / \mathrm{R}_{2}=0.3$} \\
\hline 7 & 4.6730 & 11.9584 & 20.0081 & 23.3332 & 28.6238 \\
\hline 9 & 4.6618 & 11.8998 & 19.9151 & 23.4829 & 28.3460 \\
\hline 11 & 4.6257 & 11.7400 & 19.4929 & 22.7166 & 27.5939 \\
\hline 13 & 4.6061 & 11.6458 & 19.2783 & 22.2578 & 27.2437 \\
\hline 15 & 4.5991 & 11.6232 & 19.2259 & 22.1347 & 27.1717 \\
\hline 17 & 4.5957 & 11.5982 & 19.2128 & 22.0797 & 27.1217 \\
\hline \multicolumn{6}{|c|}{ (d) $\mathrm{h} / \mathrm{R}_{2}=0.4$} \\
\hline 7 & 4.4682 & 10.8215 & 17.3870 & 20.0849 & 24.1838 \\
\hline 9 & 4.4550 & 10.7668 & 17.3148 & 20.1833 & 23.9580 \\
\hline 11 & 4.4240 & 10.6387 & 16.9951 & 19.6131 & 23.4031 \\
\hline 13 & 4.4068 & 10.5626 & 16.8302 & 19.2643 & 23.1421 \\
\hline 15 & 4.4006 & 10.5449 & 16.7894 & 19.1691 & 23.0873 \\
\hline 17 & 4.3975 & 10.5229 & 16.7787 & 19.1259 & 23.0485 \\
\hline \multicolumn{6}{|c|}{ (e) $\mathrm{h} / \mathrm{R}_{2}=0.5$} \\
\hline 7 & 4.2481 & 9.7844 & 15.2516 & 17.4708 & 20.7694 \\
\hline 9 & 4.2346 & 9.7356 & 15.1757 & 17.54 & 20.5818 \\
\hline 11 & 4.2072 & 9.6308 & 14.9252 & 17.0965 & 20.1516 \\
\hline 13 & 4.1918 & 9.5681 & 14.7947 & 16.8207 & 19.9484 \\
\hline 15 & 4.1863 & 9.5345 & 14.7619 & 16.744 & 19.9047 \\
\hline 17 & 4.1834 & 9.5345 & 14.7528 & 16.709 & 19.8732 \\
\hline
\end{tabular}

Table 4. Convergence behavior of the first five frequency parameter $\Omega=\omega R_{2}^{2} \sqrt{\rho h / D}$ for isotropic circular plate with free

\begin{tabular}{cccccc}
\hline \multicolumn{5}{c}{ boundary condition } \\
\hline $\mathrm{N}$ & \multicolumn{5}{c}{ Mode number (n, s) } \\
\cline { 2 - 6 } & $1(2,0)$ & $2(0,0)$ & $3(3,0)$ & $4(1,0)$ & $5(4,0)$ \\
\hline (a) $\mathrm{h} / \mathrm{R}_{2}=0.1$ & & & & \\
7 & 5.3340 & 8.9708 & 12.3127 & 20.2938 & 21.6057 \\
9 & 5.3287 & 8.9616 & 12.2800 & 20.2753 & 21.4854 \\
11 & 5.3043 & 8.9216 & 12.1943 & 20.0464 & 21.2480 \\
13 & 5.2816 & 8.8750 & 12.1476 & 19.9561 & 21.1588 \\
15 & 5.2808 & 8.8593 & 12.1191 & 19.8716 & 21.0441 \\
17 & 5.2505 & 8.8220 & 12.0455 & 19.7307 & 20.8762 \\
\hline
\end{tabular}


Table 4 (cont'd). Convergence behavior of the first five frequency parameter $\Omega=\omega R_{2}^{2} \sqrt{\rho h / D}$ for isotropic circular plate with free boundary condition

\begin{tabular}{cccccc}
\hline $\mathrm{N}$ & \multicolumn{5}{c}{ Mode number $(\mathrm{n}, \mathrm{s})$} \\
\cline { 2 - 5 } & $1(2,0)$ & $2(0,0)$ & $3(3,0)$ & $4(1,0)$ & $5(4,, 0)$ \\
\hline (b) $\mathrm{h} / \mathrm{R}_{2}=0.2$ & & & & \\
7 & 5.1450 & 8.5817 & 11.5491 & 18.5019 & 19.5704 \\
9 & 5.1406 & 8.5756 & 11.5380 & 18.5006 & 19.4733 \\
11 & 5.1221 & 8.5363 & 11.4515 & 18.3073 & 19.2975 \\
13 & 5.1128 & 8.5183 & 11.4210 & 18.2823 & 19.2284 \\
15 & 5.0995 & 8.4908 & 11.3541 & 18.1420 & 19.0353 \\
17 & 5.0851 & 8.4683 & 11.3080 & 18.0471 & 18.9218 \\
(c) $\mathrm{h} / \mathrm{R}_{2}=0.3$ & & & & \\
7 & 4.9285 & 8.1022 & 10.6575 & 16.5271 & 17.3937 \\
9 & 4.9250 & 8.0972 & 10.6293 & 16.5270 & 17.3172 \\
11 & 4.8947 & 8.0380 & 10.5143 & 16.2691 & 17.0355 \\
13 & 4.8888 & 8.0262 & 10.4915 & 16.2565 & 16.9841 \\
15 & 4.8773 & 8.0030 & 10.4411 & 16.1549 & 16.8300 \\
17 & 4.8641 & 7.9802 & 10.3929 & 16.0595 & \\
(d) $\mathrm{h} / \mathrm{R}_{2}=0.4$ & & & & 16.7340 \\
7 & 4.6658 & 7.5353 & 9.6766 & 14.5250 & 15.2375 \\
9 & 4.6623 & 7.5404 & 9.5458 & 14.5258 & 15.1787 \\
11 & 4.6402 & 7.4959 & 9.5760 & 14.3488 & 14.9984 \\
13 & 4.6357 & 7.4866 & 9.5581 & 14.3406 & 14.9597 \\
15 & 4.6261 & 7.4679 & 9.5210 & 14.2675 & 14.8657 \\
17 & 4.6156 & 7.4490 & 9.4833 & 14.1950 & \\
(e) $\mathrm{h} / \mathrm{R}_{2}=0.5$ & & & & 14.7787 \\
7 & 4.4078 & 7.0170 & 8.8254 & 12.8306 & 13.5307 \\
9 & 4.3946 & 6.9974 & 8.7678 & 12.7707 & 13.4038 \\
11 & 4.3823 & 6.9698 & 8.7312 & 12.6750 & 13.3186 \\
13 & 4.3736 & 6.9542 & 8.6976 & 12.6378 & 13.2480 \\
15 & 4.3657 & 6.9389 & 8.6696 & 12.5840 & 13.1788 \\
17 & 4.3560 & 6.9213 & 8.6351 & 12.5214 & \\
\hline
\end{tabular}

Table 5. Comparison of the first five frequency parameter $\Omega=\omega R_{2}^{2} \sqrt{\rho h / D}$ for isotropic circular plate with different boundary condition

\begin{tabular}{|c|c|c|c|c|c|}
\hline \multirow[t]{2}{*}{$\mathrm{h} / \mathrm{R}_{2}$} & \multicolumn{5}{|c|}{ Mode number $(\mathrm{n}, \mathrm{s})$} \\
\hline & $1(0,0)$ & $2(1,0)$ & $3(2,0)$ & $4(0,1)$ & $5(3,0)$ \\
\hline \multicolumn{6}{|c|}{ (a) Clamped circular plates } \\
\hline 0.1 & 10.0424 & 20.5753 & 33.0433 & 37.9726 & 47.3433 \\
\hline$\left[\right.$ Mindlin $\left.^{\mathrm{a}}\right]$ & 9.94 & 20.23 & 32.41 & 36.48 & - \\
\hline [3-D Ritz $\left.{ }^{\mathrm{b}}\right]$ & 9.9909 & 20.297 & 32.43 & 36.744 & 46.14 \\
\hline$\left[\right.$ Reddy's $\left.\mathrm{TPT}^{\mathrm{c}}\right]$ & 9.94614 & 20.1993 & 32.2634 & 36.5489 & 45.8905 \\
\hline 0.2 & 9.3421 & 18.1025 & 27.652 & 31.2997 & 37.8875 \\
\hline$\left[\right.$ Mindlin $\left.{ }^{\mathrm{a}}\right]$ & 9.24 & 17.83 & 27.21 & 30.21 & - \\
\hline [3-D Ritz $\left.{ }^{b}\right]$ & 9.3225 & 17.963 & 27.366 & 30.649 & 37.338 \\
\hline [Reddy's TPT ${ }^{\mathrm{c}}$ ] & 9.26503 & 17.855 & 27.2148 & 30.4749 & 37.1513 \\
\hline 0.3 & 8.4673 & 15.5135 & 22.7934 & 25.5069 & 30.3600 \\
\hline$\left[\right.$ Mindlin $\left.^{\mathrm{a}}\right]$ & 8.36 & 15.26 & 22.38 & 24.64 & - \\
\hline [3-D Ritz $\left.{ }^{b}\right]$ & 8.4676 & 15.453 & 22.667 & 25.15 & 30.093 \\
\hline [Reddy's TPT $^{\mathrm{c}}$ ] & 8.4113 & 15.385 & & 25.1011 & 30.0729 \\
\hline
\end{tabular}

\footnotetext{
${ }^{a}$ Irie et al. (1982)

${ }^{\mathrm{b}}$ Liew and Yang (1999)

${ }^{c}$ Hosseini-Hashemi et al. (2010)
} 
Table 5 (cont'd). Comparison of the first five frequency parameter $\Omega=\omega R_{2}^{2} \sqrt{\rho h / D}$ for isotropic circular plate with different boundary condition

\begin{tabular}{|c|c|c|c|c|c|}
\hline \multirow{2}{*}{$\mathrm{h} / \mathrm{R}_{2}$} & \multicolumn{5}{|c|}{ Mode number $(\mathrm{n}, \mathrm{s})$} \\
\hline & $1(0,0)$ & $2(1,0)$ & $3(2,0)$ & $4(0,1)$ & $5(3,0)$ \\
\hline 0.4 & 7.5883 & 13.2889 & 19.0545 & 21.1650 & 24.9871 \\
\hline$\left[\right.$ Mindlin $\left.{ }^{\mathrm{a}}\right]$ & 7.47 & 13.04 & 18.64 & 20.42 & - \\
\hline [3-D Ritz ${ }^{\mathrm{b}}$ ] & 7.6002 & 13.27 & 19.001 & 20.951 & 24.84 \\
\hline 0.5 & 6.7887 & 11.4950 & 16.2492 & 17.9655 & 21.1196 \\
\hline [3-D Ritz ${ }^{\mathrm{b}}$ ] & 6.8068 & 11.497 & 16.229 & 17.825 & 21.029 \\
\hline \multicolumn{6}{|c|}{ (b) Simply supported circular plates } \\
\hline 0.1 & 4.8794 & 13.5871 & 24.6232 & 28.9016 & 37.7744 \\
\hline$\left[\right.$ Mindlin $\left.^{\mathrm{a}}\right]$ & 4.89 & 13.52 & 24.41 & 28.24 & - \\
\hline [3-D Ritz $\left.{ }^{b}\right]$ & 4.8975 & 13.58 & 24.555 & 28.31 & 37.472 \\
\hline$\left[\right.$ Reddy's TPT $\left.{ }^{c}\right]$ & 4.89421 & 13.5142 & 24.3263 & 28.2547 & 36.9926 \\
\hline 0.2 & 4.7642 & 12.6999 & 21.9910 & 25.5725 & 32.2699 \\
\hline$\left[\right.$ Mindlin $\left.{ }^{\mathrm{a}}\right]$ & 4.78 & 12.67 & 21.92 & 24.99 & \\
\hline [3-D Ritz $\left.{ }^{\mathrm{b}}\right]$ & 4.7876 & 12.764 & 22.13 & 25.188 & 32.389 \\
\hline [Reddy's TPT $\left.{ }^{\mathrm{c}}\right]$ & 4.77871 & 12.6324 & 21.7279 & 25.0414 & 31.6336 \\
\hline 0.3 & 4.5991 & 11.6232 & 19.2259 & 22.1347 & 27.1717 \\
\hline$\left[\right.$ Mindlin $\left.^{\mathrm{a}}\right]$ & 4.6 & 11.6 & 19.18 & 21.59 & - \\
\hline [3-D Ritz $]$ & 4.6234 & 11.723 & 19.453 & 21.879 & - \\
\hline [Reddy's TPT ${ }^{\mathrm{c}}$ ] & 4.60704 & 11.6491 & 18.2838 & 21.6757 & 27.1569 \\
\hline 0.4 & 4.60704 & 10.5449 & 16.7894 & 19.1691 & 23.0873 \\
\hline$\left[\right.$ Mindlin $\left.{ }^{\mathrm{a}}\right]$ & 4.4006 & 10.5449 & 16.7894 & 19.1691 & 23.0873 \\
\hline [3-D Ritz $\left.{ }^{\mathrm{b}}\right]$ & 4.40 & 10.51 & 16.74 & 18.66 & - \\
\hline 0.5 & 4.6253 & 10.6620 & 17.0450 & 18.9940 & - \\
\hline [3-D Ritz $\left.{ }^{\mathrm{b}}\right]$ & 4.1863 & 9.5345 & 14.7619 & 16.744 & 19.9047 \\
\hline \multicolumn{6}{|c|}{ (c) Free circular plates } \\
\hline 0.1 & 5.2808 & 8.8593 & 12.1191 & 19.8716 & 21.0441 \\
\hline$\left[\right.$ Mindlin $\left.^{\mathrm{a}}\right]$ & 5.28 & 8.87 & - & 19.71 & - \\
\hline [3-D Ritz $\left.{ }^{\mathrm{b}}\right]$ & 5.2795 & 8.872 & 12.074 & 19.738 & 20.831 \\
\hline [Reddy's $\mathrm{TPT}^{\mathrm{c}}$ ] & 5.27842 & 8.8688 & 12.0675 & 19.7172 & - \\
\hline 0.2 & 5.0995 & 8.4908 & 11.3541 & 18.1420 & 19.0353 \\
\hline$\left[\right.$ Mindlin $\left.^{\mathrm{a}}\right]$ & 5.11 & 8.51 & - & 17.98 & - \\
\hline [3-D Ritz $\left.{ }^{\mathrm{b}}\right]$ & 5.1185 & 8.5194 & 11.337 & 18.056 & 18.882 \\
\hline [Reddy's TPT ${ }^{\mathrm{c}}$ ] & 5.11607 & 8.50842 & 11.3233 & 17.9983 & \\
\hline 0.3 & 4.8773 & 8.0030 & 10.4411 & 16.1549 & 16.8300 \\
\hline [Mindlin'] & 4.89 & 8.01 & - & 15.98 & - \\
\hline [3-D Ritz $\left.{ }^{\mathrm{b}}\right]$ & 4.9005 & 8.0344 & 10.439 & 16.102 & 16.75 \\
\hline [Reddy's TPT ${ }^{\mathrm{c}}$ ] & 4.89609 & 8.01507 & 10.4176 & 16.0153 & - \\
\hline 0.4 & 4.6261 & 7.4679 & 9.5210 & 14.2675 & 14.8657 \\
\hline$\left[\right.$ Mindlin $\left.^{\mathrm{a}}\right]$ & 4.64 & 7.46 & - & 14.09 & - \\
\hline [3-D Ritz $\left.{ }^{b}\right]$ & 4.651 & 7.5008 & 9.5294 & 14.241 & 14.806 \\
\hline 0.5 & 4.3657 & 6.9389 & 8.6696 & 12.5840 & 13.1788 \\
\hline [3-D Ritz ${ }^{\mathrm{b}}$ ] & 4.3913 & 6.9727 & 8.6854 & 12.578 & 13.145 \\
\hline
\end{tabular}

${ }^{a}$ Irie et al. (1982)

${ }^{\mathrm{b}}$ Liew and Yang (1999)

${ }^{\mathrm{c}}$ Hosseini-Hashemi et al. (2010)

The comparison studies are also carried out here in Table 5, for circular plate with different boundary conditions, in order to examine the discrepancies between the present finite element solution and Mindlin plate solution (Irie et al., 1982), 3-D Ritz 
solution (Liew and Yang, 1999) and Reddy's third-order plate theory solution (Hosseini-Hashemi et al., 2010). The comparisons show good agreement with most of differences being less than $2 \%$.

On the basis of above verification of the current approach, results of convergence behavior of the first five frequency parameters $\Omega=\omega R_{2}^{2} \sqrt{\rho h / \mathrm{D}_{o}}$ where $\mathrm{D}_{O}=\mathrm{E}_{1} \mathrm{~h}^{3} / 12\left(1-v_{12} v_{21}\right)$, for glass/epoxy laminated circular plates with thickness-radius ratios varying from 0.1 to 0.5 in step of 0.5 under different boundary condition are presented in Table 6-8. It can be seen that $\mathrm{N}=15$, is sufficient to obtain satisfactory convergence for first five frequency parameters of glass/epoxy laminated circular plates. It is seen that the first axisymmetric flexural mode $(0,0)$, is found to be lowest fundamental mode except when the boundaries of the circular plates are free, for which it is found corresponding to mode type $(2,0)$.

Table 6. Convergence of the first five frequency parameters $\Omega=\omega R_{2}^{2} \sqrt{\rho h / \mathrm{D}_{o}}$ for glass/epoxy laminated circular plate with

\begin{tabular}{|c|c|c|c|c|c|}
\hline \multirow[t]{2}{*}{$\mathrm{N}$} & \multicolumn{5}{|c|}{ Mode number $(\mathrm{n}, \mathrm{s})$} \\
\hline & $1(0,0)$ & $2(1,0)$ & $3(2,0)$ & $4(0,1)$ & $5(3,0)$ \\
\hline \multicolumn{6}{|c|}{ (a) $h / R_{2}=0.1$} \\
\hline 7 & 8.3874 & 16.3859 & 26.0089 & 31.6059 & 37.0811 \\
\hline 9 & 8.2632 & 16.0596 & 25.5660 & 31.6003 & 36.3772 \\
\hline 11 & 8.1931 & 15.8208 & 25.1543 & 30.3425 & 34.9085 \\
\hline 13 & 8.2300 & 15.7119 & 24.5106 & 29.1863 & 34.0940 \\
\hline 15 & 8.1020 & 15.9851 & 24.2491 & 28.3471 & 33.9547 \\
\hline 17 & 8.0980 & 15.7762 & 24.2969 & 28.1901 & 33.9401 \\
\hline \multicolumn{6}{|c|}{ (b) $h / R_{2}=0.2$} \\
\hline 7 & 7.4086 & 13.4218 & 19.9487 & 23.3156 & 26.3882 \\
\hline 9 & 7.2497 & 13.0520 & 19.5856 & 23.3176 & 25.7000 \\
\hline 11 & 7.2543 & 13.0372 & 19.3658 & 22.6315 & 25.4753 \\
\hline 13 & 7.3078 & 13.03199 & 19.1947 & 22.2186 & 25.1737 \\
\hline 15 & 7.1669 & 13.1066 & 18.9193 & 21.6903 & 25.2061 \\
\hline 17 & 7.1586 & 13.0356 & 18.9016 & 21.5810 & 25.0928 \\
\hline \multicolumn{6}{|c|}{ (c) $h / R_{2}=0.3$} \\
\hline 7 & 6.3889 & 10.9119 & 15.5775 & 17.808 & 19.8686 \\
\hline 9 & 6.2320 & 10.5662 & 15.3035 & 17.8741 & 19.7728 \\
\hline 11 & 6.2713 & 10.6475 & 15.1564 & 17.3843 & 19.3774 \\
\hline 13 & 6.3247 & 10.6737 & 15.1200 & 17.2210 & 19.2303 \\
\hline 15 & 6.1890 & 10.6305 & 14.9014 & 16.8999 & 19.3194 \\
\hline 17 & 6.1706 & 10.6149 & 14.8636 & 16.7987 & 19.1709 \\
\hline \multicolumn{6}{|c|}{ (d) $h / R_{2}=0.4$} \\
\hline 7 & 5.4987 & 9.0334 & 12.604 & 14.2456 & 15.793 \\
\hline 9 & 5.3552 & 8.7255 & 12.3986 & 14.3448 & 15.7495 \\
\hline 11 & 5.4074 & 8.8372 & 12.2893 & 13.946 & 15.4773 \\
\hline 13 & 5.4564 & 8.8717 & 12.2812 & 13.8732 & 15.3801 \\
\hline 15 & 5.3342 & 8.7836 & 12.1203 & 13.6708 & 15.4904 \\
\hline 17 & 5.3084 & 8.7838 & 12.0818 & 13.5726 & 15.3416 \\
\hline \multicolumn{6}{|c|}{ (e) $h / R_{2}=0.5$} \\
\hline 7 & 4.7716 & 7.6485 & 10.5269 & 11.8264 & 13.0627 \\
\hline 9 & 4.6436 & 7.3764 & 10.3698 & 11.9373 & 13.0384 \\
\hline 11 & 4.6981 & 7.4922 & 10.2816 & 11.5932 & 12.8352 \\
\hline 13 & 4.7421 & 7.5277 & 10.2824 & 11.5576 & 12.7599 \\
\hline 15 & 4.6340 & 7.4270 & 10.1593 & 11.4264 & 12.9467 \\
\hline 17 & 4.6050 & 7.4361 & 10.1237 & 11.3309 & 12.7340 \\
\hline
\end{tabular}


Table 7. Convergence of the first five frequency parameters $\Omega=\omega R_{2}^{2} \sqrt{\rho h / \mathrm{D}_{o}}$ for glass/epoxy laminated circular plate with simply supported boundary condition $\left(\beta=30^{\circ}\right)$

\begin{tabular}{|c|c|c|c|c|c|}
\hline \multirow[t]{2}{*}{$\mathrm{N}$} & \multicolumn{5}{|c|}{ Mode number $(\mathrm{n}, \mathrm{s})$} \\
\hline & $1(0,0)$ & $2(1,0)$ & $3(2,0)$ & $4(0,1)$ & $5(3,0)$ \\
\hline \multicolumn{6}{|c|}{ (a) $\mathrm{h} / \mathrm{R}_{2}=0.1$} \\
\hline 7 & 3.5034 & 10.4407 & 18.7796 & 24.2754 & 28.9114 \\
\hline 9 & 3.5393 & 10.1735 & 18.5739 & 24.0317 & 28.4791 \\
\hline 11 & 3.4383 & 10.0711 & 18.3614 & 23.4164 & 27.3723 \\
\hline 13 & 3.3718 & 9.9909 & 17.9813 & 22.9590 & 26.8351 \\
\hline 15 & 3.4045 & 9.9820 & 17.4809 & 21.8198 & 26.5979 \\
\hline 17 & 3.4005 & 9.8816 & 17.4502 & 21.6320 & 26.4817 \\
\hline \multicolumn{6}{|c|}{ (b) $\mathrm{h} / \mathrm{R}_{2}=0.2$} \\
\hline 7 & 3.3513 & 9.3009 & 15.6748 & 19.6000 & 22.3177 \\
\hline 9 & 3.3618 & 9.0275 & 15.4348 & 19.4544 & 22.0991 \\
\hline 11 & 3.2802 & 8.9684 & 15.2882 & 19.0893 & 21.5911 \\
\hline 13 & 3.2426 & 8.9554 & 15.1415 & 18.8529 & 21.2247 \\
\hline 15 & 3.2616 & 8.9813 & 14.7796 & 18.1214 & 21.2389 \\
\hline 17 & 3.2673 & 8.8973 & 14.7225 & 17.9959 & 21.0709 \\
\hline \multicolumn{6}{|c|}{ (c) $h / R_{2}=0.3$} \\
\hline 7 & 3.1866 & 8.1463 & 12.9774 & 15.7974 & 17.5112 \\
\hline 9 & 3.1831 & 7.8931 & 12.7677 & 15.7920 & 17.4032 \\
\hline 11 & 3.1188 & 7.8659 & 12.6444 & 15.4911 & 17.1239 \\
\hline 13 & 3.0993 & 7.8798 & 12.5900 & 15.3575 & 16.9039 \\
\hline 15 & 3.1057 & 7.9357 & 12.3547 & 14.9203 & 17.0321 \\
\hline 17 & 3.1145 & 7.8662 & 12.2868 & 14.8204 & 16.8499 \\
\hline \multicolumn{6}{|c|}{ (d) $h / R_{2}=0.4$} \\
\hline 7 & 3.0081 & 7.1210 & 10.8856 & 12.9907 & 14.2142 \\
\hline 9 & 2.9944 & 6.8871 & 10.7105 & 13.0686 & 14.1535 \\
\hline 11 & 2.9469 & 6.8832 & 10.6032 & 12.7802 & 13.9926 \\
\hline 13 & 2.9399 & 6.9064 & 10.5749 & 12.6992 & 13.852 \\
\hline 15 & 2.937 & 6.9611 & 10.4322 & 12.4442 & 14.0017 \\
\hline 17 & 2.9456 & 6.9219 & 10.3624 & 12.3672 & 13.8266 \\
\hline \multicolumn{6}{|c|}{ (e) $h / R_{2}=0.5$} \\
\hline 7 & 2.8262 & 6.2571 & 9.2906 & 10.8330 & 11.8976 \\
\hline 9 & 2.8050 & 6.0404 & 9.1440 & 10.9657 & 11.7916 \\
\hline 11 & 2.7721 & 6.0522 & 9.0509 & 10.6362 & 11.7220 \\
\hline 13 & 2.7741 & 6.0779 & 9.0339 & 10.5755 & 11.6313 \\
\hline 15 & 2.7646 & 6.1150 & 8.9441 & 10.5011 & 11.7325 \\
\hline 17 & 2.7716 & 6.1084 & 8.8769 & 10.4702 & 11.6151 \\
\hline
\end{tabular}

Table 8. Convergence of the first five frequency parameter $\Omega=\omega R_{2}^{2} \sqrt{\rho h / \mathrm{D}_{o}}$ for glass/epoxy laminated circular plate with free boundary condition $\left(\beta=30^{\circ}\right)$

\begin{tabular}{crrrrc}
\hline $\mathrm{N}$ & \multicolumn{5}{c}{ Mode number $(\mathrm{n}, \mathrm{s})$} \\
\cline { 2 - 6 } & $1(2,0)$ & $2(0,0)$ & $3(3,0)$ & $4(1,0)$ & $5(4,0)$ \\
\hline 7 & $(\mathrm{a}) \mathrm{h} / \mathrm{R}_{2}=0.1$ & & & & \\
9 & 3.7118 & 6.3054 & 8.3146 & 14.1627 & 15.2148 \\
11 & 3.7708 & 6.3018 & 8.3498 & 14.1136 & 15.1682 \\
13 & 3.6918 & 6.2431 & 8.2231 & 13.8570 & 14.6622 \\
15 & 3.8405 & 6.4455 & 8.5263 & 14.1537 & 14.5310 \\
17 & 3.8022 & 6.3160 & 8.5008 & 13.8973 & 14.3509 \\
& 3.7130 & 6.2030 & 8.2279 & 13.7343 & 14.2021 \\
\hline
\end{tabular}


Table 8 (cont'). Convergence of the first five frequency parameter $\Omega=\omega R_{2}^{2} \sqrt{\rho h / \mathrm{D}_{o}}$ for glass/epoxy laminated circular plate with free boundary condition $\left(\beta=30^{\circ}\right)$

\begin{tabular}{cccccc}
\hline $\mathrm{N}$ & \multicolumn{5}{c}{ Mode number $(\mathrm{n}, \mathrm{s})$} \\
\cline { 2 - 5 } & $1(2,0)$ & $2(0,0)$ & $3(3,0)$ & $4(1,0)$ & $5(4,0)$ \\
\hline & $(\mathrm{b}) \mathrm{h} / \mathrm{R}_{2}=0.2$ & & & \\
9 & 3.5401 & 5.9275 & 7.6237 & 12.399 & 13.2206 \\
11 & 3.5964 & 5.9130 & 7.6529 & 12.3433 & 13.2208 \\
13 & 3.5290 & 5.8743 & 7.5478 & 12.1656 & 12.7929 \\
15 & 3.6725 & 6.0055 & 7.8013 & 12.4383 & 12.7138 \\
17 & 3.6219 & 5.8814 & 7.7490 & 12.0854 & 12.5366 \\
& 3.5508 & 5.8334 & 7.5242 & 12.1015 & \\
7 & $(\mathrm{c}) \mathrm{h} / \mathrm{R}_{2}=0.3$ & & & & 11.2215 \\
9 & 3.3556 & 5.4576 & 6.8876 & 10.7073 & 11.2743 \\
11 & 3.4104 & 5.4394 & 6.9090 & 10.6440 & 10.7682 \\
13 & 3.3337 & 5.3931 & 6.7555 & 10.3785 & 10.8197 \\
15 & 3.4643 & 5.4529 & 6.956 & 10.6026 & 10.7760 \\
17 & 3.4040 & 5.4097 & 6.9121 & 10.4271 & \\
& 3.3517 & 5.3347 & 6.7305 & 10.3446 & 9.4061 \\
7 & $(\mathrm{~d}) \mathrm{h} / \mathrm{R}_{2}=0.4$ & & & & 9.4942 \\
9 & 3.1239 & 5.0220 & 6.0971 & 9.1122 & 9.1032 \\
11 & 3.1699 & 5.0015 & 6.1170 & 9.0530 & 9.2664 \\
13 & 3.1140 & 4.9831 & 6.0078 & 8.8975 & 9.1244 \\
15 & 3.2316 & 4.9889 & 6.1693 & 9.0876 & \\
17 & 3.1753 & 4.9471 & 6.1417 & 8.9397 & 8.0256 \\
& 3.1310 & 4.9117 & 5.9955 & 8.897 & 7.77775 \\
7 & $(\mathrm{e}) \mathrm{h} / \mathrm{R}_{2}=0.5$ & & & & 7.8531 \\
9 & 2.9136 & 4.6334 & 5.4695 & 7.9306 & 7.7679 \\
11 & 2.9419 & 4.5941 & 5.4415 & 7.9438 & 7.8339 \\
13 & 2.9052 & 4.6010 & 5.3842 & 7.8039 & 7.7262 \\
15 & 3.0032 & 4.5626 & 5.4994 & 7.6938 & \\
17 & 2.9521 & 4.5218 & 5.4795 & 5.3539 & \\
\hline
\end{tabular}

Table 9. Effect of varying fiber angle $\beta$ and thickness-radius ratios $\mathrm{h} / \mathrm{R}_{2}$, on frequency parameter $\Omega=\omega R_{2}^{2} \sqrt{\rho h / \mathrm{D}_{o}}$ for glass/epoxy laminated circular plate with clamped boundary condition.

\begin{tabular}{ccccccc}
\hline $\mathrm{h} / \mathrm{R}_{2}$ & $\beta$ & \multicolumn{5}{c}{ Mode number $(\mathrm{n}, \mathrm{s})$} \\
\cline { 2 - 6 } & & $1(0,0)$ & $2(1,0)$ & $3(2,0)$ & $4(0,1)$ & $5(3,0)$ \\
\hline 0.1 & $0^{\circ}$ & 8.5546 & 16.6147 & 24.7524 & 29.4499 & 34.1353 \\
& $15^{\circ}$ & 8.5299 & 16.5515 & 24.7900 & 29.3319 & 34.1970 \\
& $30^{\circ}$ & 8.1020 & 15.9852 & 24.2492 & 28.3472 & 33.9548 \\
& $45^{\circ}$ & 7.4637 & 15.02533 & 23.4767 & 26.9270 & 33.388 \\
& $60^{\circ}$ & 6.9082 & 14.1306 & 22.7472 & 25.5591 & 32.5613 \\
& $75^{\circ}$ & 6.6518 & 13.5807 & 22.1579 & 24.6051 & 31.6929 \\
0.2 & $90^{\circ}$ & 6.6224 & 13.2927 & 21.7162 & 24.1544 & 31.1096 \\
\hline & $0^{\circ}$ & 7.5677 & 13.5866 & 19.2229 & 22.5626 & 25.3377 \\
& $15^{\circ}$ & 7.5332 & 13.5207 & 19.2428 & 22.4558 & 25.4063 \\
& $30^{\circ}$ & 7.1669 & 13.1066 & 18.9193 & 21.6903 & 25.2061 \\
& $45^{\circ}$ & 6.6354 & 12.4673 & 18.3603 & 20.5426 & 24.6637 \\
& $60^{\circ}$ & 6.1516 & 11.7058 & 17.6803 & 19.3599 & 23.9775 \\
& $75^{\circ}$ & 5.8736 & 11.1019 & 17.0165 & 18.4299 & 23.3043 \\
& $90^{\circ}$ & 5.7931 & 10.7894 & 16.5504 & 17.9479 & 22.8703 \\
\hline
\end{tabular}


Table 9 (cont'd). Effect of varying fiber angle $\beta$ and thickness-radius ratios $\mathrm{h} / \mathrm{R}_{2}$, on frequency parameter $\Omega=\omega R_{2}^{2} \sqrt{\rho h / \mathrm{D}_{o}}$ for glass/epoxy laminated circular plate with clamped boundary condition.

\begin{tabular}{ccccccc}
\hline $\mathrm{h} / \mathrm{R}_{2}$ & $\beta$ & \multicolumn{5}{c}{ Mode number $(\mathrm{n}, \mathrm{s})$} \\
\cline { 2 - 6 } & & $1(0,0)$ & $2(1,0)$ & $3(2,0)$ & $4(0,1)$ & $5(3,0)$ \\
\hline 0.3 & $0^{\circ}$ & 6.5123 & 10.9856 & 15.0768 & 17.5489 & 19.4125 \\
& $15^{\circ}$ & 6.4805 & 10.9345 & 15.1021 & 17.4743 & 19.4798 \\
& $30^{\circ}$ & 6.1890 & 10.6305 & 14.9014 & 16.8999 & 19.3194 \\
& $45^{\circ}$ & 5.7616 & 10.1624 & 14.4885 & 15.9917 & 18.8902 \\
& $60^{\circ}$ & 5.3437 & 9.5605 & 13.9163 & 15.0161 & 18.3667 \\
& $75^{\circ}$ & 5.0536 & 8.9877 & 13.3223 & 14.2201 & 17.8882 \\
& $90^{\circ}$ & 4.9334 & 8.6585 & 12.9252 & 13.7989 & 17.6097 \\
\hline .4 & $0^{\circ}$ & 5.5909 & 9.0584 & 12.2336 & 14.1727 & 15.5552 \\
& $15^{\circ}$ & 5.5647 & 9.0186 & 12.2614 & 14.1189 & 15.6194 \\
& $30^{\circ}$ & 5.3342 & 8.7836 & 12.1203 & 13.6708 & 15.4904 \\
& $45^{\circ}$ & 4.9882 & 8.4219 & 11.7995 & 12.9381 & 15.1474 \\
& $60^{\circ}$ & 4.6260 & 7.9337 & 11.3289 & 12.1335 & 14.7456 \\
& $75^{\circ}$ & 4.3411 & 7.4262 & 10.8364 & 11.4702 & 14.4052 \\
& $90^{\circ}$ & 4.2024 & 7.1142 & 10.5202 & 11.1163 & 14.2314 \\
\hline & $0^{\circ}$ & 4.8415 & 7.6519 & 10.2403 & 11.8281 & 13.0692 \\
& $15^{\circ}$ & 4.8201 & 7.6184 & 10.2673 & 11.7882 & 12.9801 \\
& $30^{\circ}$ & 4.634 & 7.427 & 10.1513 & 11.4264 & 12.8757 \\
& $45^{\circ}$ & 4.3477 & 7.1339 & 9.8978 & 10.8194 & 12.5935 \\
& $60^{\circ}$ & 4.0316 & 6.7284 & 9.5076 & 10.1438 & 12.2749 \\
& $75^{\circ}$ & 3.7623 & 6.287 & 9.1029 & 9.5838 & 12.0243 \\
& $90^{\circ}$ & 3.6149 & 6.0588 & 8.8514 & 9.284 & 11.9136 \\
\hline
\end{tabular}

Table 9 shows the effect of varying fiber angle $\beta$ and thickness-radius ratios $h / R_{2}$ on frequency parameters $\Omega=\omega R_{2}^{2} \sqrt{\rho h / \mathrm{D}_{o}}$, for glass/epoxy laminated circular plate with clamped boundary condition. It is observed that increasing the fiber orientation angle from $0^{\circ}$ to $90^{\circ}$ decreases the frequency parameters. This effect is larger as the thicknessradius ratios increases. The frequency parameters is observed to be maximum at fiber angle $\beta=0^{\circ}$.The differences of fundamental frequencies between fiber angle $0^{\circ}$ and $90^{\circ}$ are approximately $22.58 \%, 23.44 \%, 24.24 \%, 24.83 \%$,and $27.65 \%$ for $\mathrm{h} / \mathrm{R}_{2}$ $=0.1,0.2,0.3,0.4$ and 0.5 respectively. As seen, as the thickness-radius ratios increases, the differences of fundamental frequencies increased.

Table 10. Effect of varying fiber angle $\beta$ and thickness-radius ratios $h / \mathrm{R}_{2}$, on frequency parameter $\Omega=\omega R_{2}^{2} \sqrt{\rho h / \mathrm{D}_{o}}$ for glass/epoxy laminated circular plate with simply supported boundary condition.

\begin{tabular}{ccccccc}
\hline $\mathrm{h} / \mathrm{R}_{2}$ & $\beta$ & \multicolumn{5}{c}{ Mode number $(\mathrm{n}, \mathrm{s})$} \\
\cline { 2 - 6 } & & $1(0,0)$ & $2(1,0)$ & $3(2,0)$ & $4(0,1)$ & $5(3,0)$ \\
\hline 0.1 & $0^{\circ}$ & 3.3775 & 10.0885 & 17.4190 & 22.4934 & 26.2291 \\
& $15^{\circ}$ & 3.3778 & 10.0459 & 17.4016 & 22.4156 & 26.2910 \\
& $30^{\circ}$ & 3.4045 & 9.9820 & 17.481 & 21.8198 & 26.5979 \\
& $45^{\circ}$ & 3.4758 & 9.9278 & 17.6586 & 20.9956 & 26.9934 \\
& $60^{\circ}$ & 3.5998 & 9.8639 & 17.7603 & 20.1939 & 27.1412 \\
& $75^{\circ}$ & 3.7438 & 9.705 & 17.5563 & 19.5900 & 26.8920 \\
& $90^{\circ}$ & 3.8368 & 9.4683 & 17.1876 & 19.2662 & 26.5024 \\
\hline
\end{tabular}


Table 10 (cont'd). Effect of varying fiber angle $\beta$ and thickness-radius ratios $\mathrm{h} / \mathrm{R}_{2}$, on frequency parameter $\Omega=\omega R_{2}^{2} \sqrt{\rho h / \mathrm{D}_{o}}$ for glass/epoxy laminated circular plate with simply supported boundary condition.

\begin{tabular}{|c|c|c|c|c|c|c|}
\hline \multirow[t]{2}{*}{$\mathrm{h} / \mathrm{R}_{2}$} & \multirow[t]{2}{*}{$\beta$} & \multicolumn{5}{|c|}{ Mode number $(n, s)$} \\
\hline & & $1(0,0)$ & $2(1,0)$ & $3(2,0)$ & $4(0,1)$ & $5(3,0)$ \\
\hline \multirow[t]{7}{*}{0.2} & $0^{\circ}$ & 3.2695 & 9.0967 & 14.7802 & 18.8029 & 21.0527 \\
\hline & $15^{0}$ & 3.2591 & 9.0653 & 14.7761 & 18.6957 & 21.1152 \\
\hline & $30^{\circ}$ & 3.2616 & 8.9813 & 14.7796 & 18.1214 & 21.2389 \\
\hline & $45^{\circ}$ & 3.3075 & 8.8860 & 14.8105 & 17.3512 & 21.3199 \\
\hline & $60^{\circ}$ & 3.4045 & 8.7811 & 14.8079 & 16.6381 & 21.3604 \\
\hline & $75^{0}$ & 3.5264 & 8.6327 & 14.6720 & 16.1170 & 21.3039 \\
\hline & $90^{\circ}$ & 3.6140 & 8.4573 & 14.4608 & 15.8404 & 21.1842 \\
\hline \multirow[t]{7}{*}{0.3} & $0^{\circ}$ & 3.1339 & 8.0235 & 12.3738 & 15.4776 & 16.9228 \\
\hline & $15^{0}$ & 3.1195 & 8.0141 & 12.3794 & 15.39 & 16.9834 \\
\hline & $30^{\circ}$ & 3.1057 & 7.9357 & 12.3547 & 14.9203 & 17.0321 \\
\hline & $45^{\circ}$ & 3.1253 & 7.8172 & 12.3188 & 14.2497 & 17.0024 \\
\hline & $60^{\circ}$ & 3.1866 & 7.6798 & 12.257 & 13.6087 & 16.9916 \\
\hline & $75^{0}$ & 3.2719 & 7.5225 & 12.1342 & 13.1368 & 16.9834 \\
\hline & $90^{\circ}$ & 3.3392 & 7.3875 & 11.9952 & 12.8931 & 16.9619 \\
\hline \multirow[t]{7}{*}{0.4} & $0^{\circ}$ & 2.9792 & 7.0389 & 10.4565 & 12.8915 & 13.9258 \\
\hline & $15^{0}$ & 2.9636 & 7.0355 & 10.4651 & 12.8269 & 14.1596 \\
\hline & $30^{\circ}$ & 2.9370 & 6.9611 & 10.4322 & 12.4442 & 14.0017 \\
\hline & $45^{\circ}$ & 2.9323 & 6.8486 & 10.3735 & 11.8704 & 13.9461 \\
\hline & $60^{\circ}$ & 2.9597 & 6.7062 & 10.2885 & 11.3056 & 13.9137 \\
\hline & $75^{0}$ & 3.0090 & 6.5418 & 10.1688 & 10.8876 & 13.9080 \\
\hline & $90^{\circ}$ & 3.0536 & 6.4202 & 10.0600 & 10.6771 & 13.9157 \\
\hline \multirow[t]{7}{*}{0.5} & $0^{\circ}$ & 2.8171 & 6.1966 & 8.9676 & 10.9020 & 11.8000 \\
\hline & $15^{0}$ & 2.8015 & 6.1885 & 8.9760 & 10.8693 & 11.8186 \\
\hline & $30^{\circ}$ & 2.7646 & 6.1150 & 8.9441 & 10.5011 & 11.7941 \\
\hline & $45^{0}$ & 2.7393 & 6.0132 & 8.8825 & 10.0217 & 11.7341 \\
\hline & $60^{\circ}$ & 2.7382 & 5.8898 & 8.7927 & 9.5553 & 11.6953 \\
\hline & $75^{0}$ & 2.7577 & 5.7244 & 8.6749 & 9.2076 & 11.6832 \\
\hline & $90^{\circ}$ & 2.7827 & 5.6046 & 8.5798 & 9.0324 & 11.7000 \\
\hline
\end{tabular}

Table 10 shows the effect of varying fiber angle $\beta$ and thickness-radius ratios $h / R_{2}$ on frequency parameters $\Omega=\omega R_{2}^{2} \sqrt{\rho h / \mathrm{D}_{o}}$, for glass/epoxy laminated circular plate with simply supported boundary condition. The fundamental frequency parameters are observed to be maximum at fiber angle $\beta=0^{\circ}$. It is also observed that with an increase in fiber angle from $0^{\circ}$ to $90^{\circ}$, it increases monotonically for thickness to radius ratio $h / \mathrm{R}_{2}=0.1$ and it decrease for the fiber angle $0^{\circ}$ to $15^{\circ}, 0^{\circ}$ to $30^{\circ}, 0^{\circ}$ to $45^{\circ}$ and $0^{\circ}$ to $60^{\circ}$, for thickness-radius ratios $\mathrm{h} / \mathrm{R}_{2}=0.2,0.3,0.4$ and 0.5 respectively and increases it afterward. The differences of fundamental frequencies between fiber angle $0^{\circ}$ and $90^{\circ}$ are approximately $13.59 \%, 10.53 \%, 6.55 \%, 2.49 \%$, and $1.22 \%$ for $\mathrm{h} / \mathrm{R}_{2}=0.1,0.2,0.3,0.4$ and 0.5 respectively. As seen, as the thickness-radius ratios increases, the differences of fundamental frequencies decreases. The second $(1,0)$ and fourth $(0,1)$ frequency parameters decreases with an increase in fiber orientation angle from $0^{\circ}$ to $90^{\circ}$. For thickness to radius ratios 0.1 and 0.2 , third $(2,0)$ frequency parameters first decreases and then increases a little bit and then again deceases as the fiber angle increases. The fifth $(3,0)$ frequency parameters first increases and then, again decreases as the fiber angle increased. 
Table 11. Effect of varying fiber angle $\beta$ and thickness-radius ratios $\mathrm{h} / \mathrm{R}_{2}$, on frequency parameter $\Omega=\omega R_{2}^{2} \sqrt{\rho h / \mathrm{D}_{o}}$ for glass/epoxy laminated circular plate with free boundary condition.

\begin{tabular}{|c|c|c|c|c|c|c|}
\hline \multirow[t]{2}{*}{$\mathrm{h} / \mathrm{R}_{2}$} & \multirow[t]{2}{*}{$\beta$} & \multicolumn{5}{|c|}{ Mode number $(\mathrm{n}, \mathrm{s})$} \\
\hline & & $1(2,0)$ & $2(0,0)$ & $3(3,0)$ & $4(4,0)$ & $5(1,0)$ \\
\hline \multirow[t]{7}{*}{0.1} & $0^{\circ}$ & 3.7547 & 6.1852 & 8.3753 & 13.9372 & 14.6177 \\
\hline & $15^{0}$ & 3.7616 & 6.2356 & 8.3823 & 13.8973 & 14.4368 \\
\hline & $30^{\circ}$ & 3.8022 & 6.3160 & 8.5008 & 14.3509 & 13.9222 \\
\hline & $45^{\circ}$ & 3.8834 & 6.4220 & 8.7275 & 14.7152 & 13.7542 \\
\hline & $60^{\circ}$ & 4.0074 & 6.5759 & 9.1161 & 15.5451 & 13.7643 \\
\hline & $75^{0}$ & 4.1613 & 6.7662 & 9.6596 & 16.7786 & 14.0125 \\
\hline & $90^{\circ}$ & 4.2842 & 6.8771 & 10.1566 & 17.9789 & 14.3517 \\
\hline \multirow[t]{7}{*}{0.2} & $0^{\circ}$ & 3.5743 & 5.8201 & 7.6320 & 12.1120 & 12.7627 \\
\hline & $15^{0}$ & 3.5816 & 5.8315 & 7.6404 & 12.0854 & 12.6293 \\
\hline & $30^{\circ}$ & 3.6219 & 5.8814 & 7.7490 & 12.5366 & 12.1984 \\
\hline & $45^{\circ}$ & 3.6964 & 5.9788 & 7.9504 & 12.7820 & 12.1701 \\
\hline & $60^{\circ}$ & 3.8063 & 6.1079 & 8.2836 & 13.4542 & 12.1612 \\
\hline & $75^{0}$ & 3.9501 & 6.2545 & 8.7677 & 14.4887 & 12.2446 \\
\hline & $90^{\circ}$ & 4.0797 & 6.3611 & 9.2436 & 15.5233 & 12.4988 \\
\hline \multirow[t]{7}{*}{0.3} & $0^{\circ}$ & 3.3600 & 5.4015 & 6.8178 & 10.3369 & 10.8976 \\
\hline & $15^{0}$ & 3.3654 & 5.3881 & 6.8191 & 10.3108 & 10.8301 \\
\hline & $30^{\circ}$ & 3.4040 & 5.4097 & 6.9121 & 10.7760 & 10.4271 \\
\hline & $45^{\circ}$ & 3.4734 & 5.4859 & 7.09900 & 10.9239 & 10.5106 \\
\hline & $60^{\circ}$ & 3.5737 & 5.5857 & 7.3970 & 11.4726 & 10.5146 \\
\hline & $75^{0}$ & 3.7060 & 5.6900 & 7.8155 & 12.3138 & 10.5782 \\
\hline & $90^{\circ}$ & 3.8309 & 5.7859 & 8.2271 & 13.1354 & 10.6585 \\
\hline \multirow[t]{7}{*}{0.4} & $0^{\circ}$ & 3.1354 & 4.978 & 6.0656 & 8.8684 & 9.2407 \\
\hline & $15^{0}$ & 3.1391 & 4.949 & 6.0603 & 8.8399 & 9.2355 \\
\hline & $30^{\circ}$ & 3.1753 & 4.9471 & 6.1417 & 9.2477 & 8.9397 \\
\hline & $45^{\circ}$ & 3.241 & 4.9992 & 6.3139 & 9.3812 & 9.0447 \\
\hline & $60^{\circ}$ & 3.3348 & 5.0734 & 6.5843 & 9.8441 & 9.0638 \\
\hline & $75^{0}$ & 3.4565 & 5.1504 & 6.9473 & 10.5376 & 9.0788 \\
\hline & $90^{\circ}$ & 3.5703 & 5.2372 & 7.2928 & 11.1912 & 9.0887 \\
\hline \multirow[t]{7}{*}{0.5} & $0^{\circ}$ & 2.9165 & 4.5782 & 5.419 & 7.6801 & 7.963 \\
\hline & $15^{0}$ & 2.9187 & 4.541 & 5.4089 & 7.6539 & 7.896 \\
\hline & $30^{\circ}$ & 2.9521 & 4.5218 & 5.4795 & 7.9618 & 7.7262 \\
\hline & $45^{\circ}$ & 3.0135 & 4.5514 & 5.6351 & 8.1441 & 7.8167 \\
\hline & $60^{\circ}$ & 3.101 & 4.605 & 5.8805 & 8.553 & 7.8491 \\
\hline & $75^{0}$ & 3.2129 & 4.6654 & 6.1995 & 9.1381 & 7.839 \\
\hline & $90^{\circ}$ & 3.3153 & 4.7458 & 6.4917 & 9.6727 & 7.8082 \\
\hline
\end{tabular}

Table 11 shows the effect of varying fiber angle $\beta$ and thickness-radius ratios $h / \mathrm{R}_{2}$ on frequency parameters $\Omega=\omega R_{2}^{2} \sqrt{\rho h / \mathrm{D}_{O}}$, for glass/epoxy laminated circular plate with free boundary condition. It is observed increasing the fiber orientation angle from $0^{\circ}$ to $90^{\circ}$ increases the frequency parameters. The fundamental frequency parameters $(2,0)$ are observed to be maximum at fiber angle $\beta=90^{\circ}$. The differences of fundamental frequencies between fiber angle $0^{\circ}$ and $90^{\circ}$ are approximately $14.10 \%, 14.13 \%, 4.01 \%, 13.87 \%$, and $13.67 \%$ for $\mathrm{h} / \mathrm{R}_{2} \quad=0.1,0.2,0.3,0.4$ and 0.5 respectively. As seen, as the thickness-radius ratios increases, the differences of fundamental frequencies decreases. The second $(0,0)$ frequency parameters increase as the fiber orientation angle increases from $0^{\circ}$ to $90^{\circ}$, for thickness-radius ratios $h / R_{2}=0.1$ and 0.2 whereas, for $\mathrm{h} / \mathrm{R}_{2}=0.3,0.4$ and 0.5 , it first decreased and then increases as the fiber orientation angle increases from $0^{\circ}$ to 90 . 
It is noticed that the behavior of third $(3,0)$ frequency parameters remains the same as that of the fundamental frequency parameters, i.e. the frequencies parameters increases as the fiber orientation angle increases from $0^{\circ}$ to $90^{\circ}$. The fourth $(4,0)$ and fifth $(1,0)$ frequency parameters first decreased and then again, increases as the fiber orientation angle increases from $0^{\circ}$ to $90^{\circ}$. Also, the first five natural modes, for the glass/epoxy laminated circular plates with clamp, simply supported and free boundary condition are shown in figure 4-6.

Table 12.The first five natural modes, for the glass/epoxy laminated circular plates with clamp boundary condition $\left(\beta=30^{\circ}\right)$.

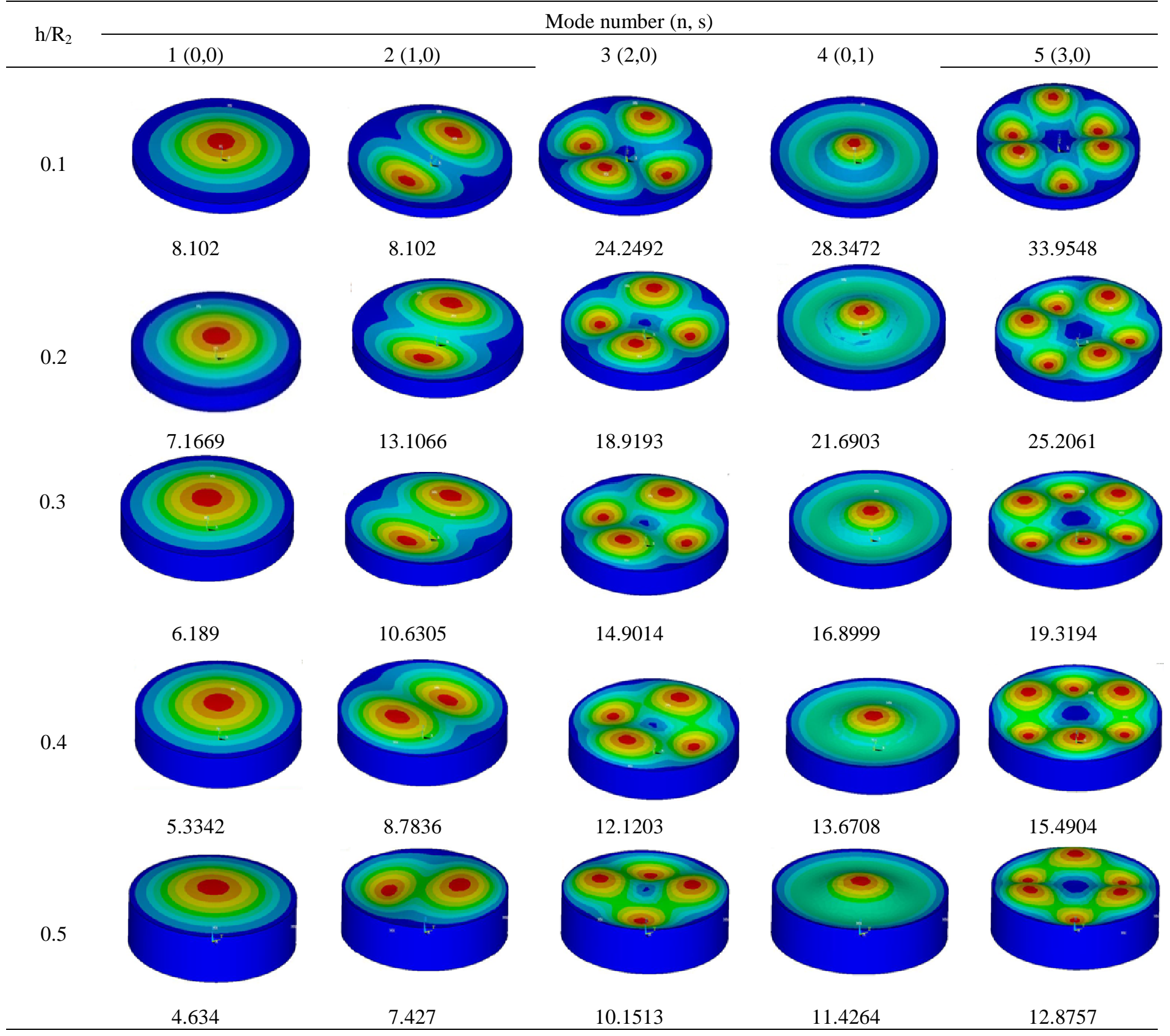


Table 13.The first five natural modes, for the glass/epoxy laminated circular plates with simply supported boundary condition

\begin{tabular}{|c|c|c|c|c|c|}
\hline \multirow{2}{*}{$\mathrm{h} / \mathrm{R}_{2}$} & \multicolumn{5}{|c|}{ Mode number $(\mathrm{n}, \mathrm{s})$} \\
\hline & $1(0,0)$ & $2(1,0)$ & $3(2,0)$ & $4(0,1)$ & $5(3,0)$ \\
\hline \multicolumn{6}{|l|}{0.1} \\
\hline & 3.4045 & 9.982 & 17.481 & 21.8198 & 26.5979 \\
\hline \multicolumn{6}{|l|}{0.2} \\
\hline & 3.2616 & 8.9813 & 14.7796 & 18.1214 & 21.2389 \\
\hline \multicolumn{6}{|l|}{0.3} \\
\hline & 3.1057 & 7.9357 & 12.3547 & 14.9203 & 17.0321 \\
\hline \multicolumn{6}{|l|}{0.4} \\
\hline & 2.937 & 6.9611 & 10.4322 & 12.4442 & 14.0017 \\
\hline \multicolumn{6}{|l|}{0.5} \\
\hline & 2.7646 & 6.115 & 8.9441 & 10.5011 & 11.7941 \\
\hline
\end{tabular}


Table 14. The first five natural modes, for the glass/epoxy laminated circular plates with free boundary condition $\left(\beta=30^{\circ}\right)$.

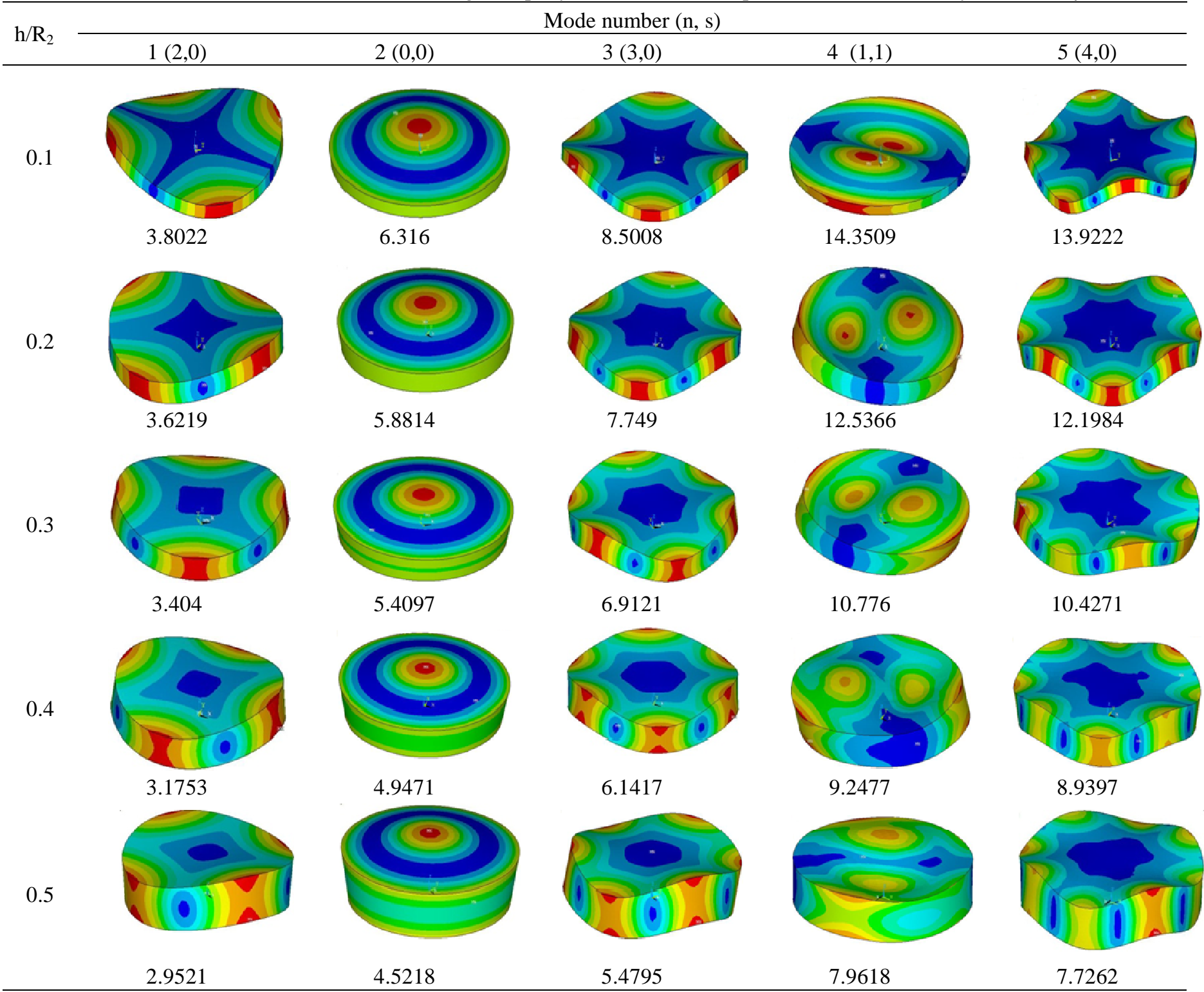

\section{Conclusions}

In this paper, a three-dimensional finite element analysis is employed for the free vibration of thick laminated composite circular plates with clamp, simply supported and free boundary condition. The effect of fiber orientation angle, thickness-radius ratios and boundary conditions on the convergence behavior was fully investigated. After comparing the present solution with the threedimensional Ritz solution, two-dimensional Mindlin plate solution and Reddy's third-order plate theory solution in the literature, free vibration behavior of thick laminated composite circular plates with various thickness-radius ratios was investigated. Also, first five vibration modes for different boundary conditions are shown in pictorial forms. For clamped laminated composite circular plates, fundamental frequency parameters are observed to be maximum at fiber angle $\beta=0^{\circ}$. The fundamental frequency parameters for both simply supported and free laminated circular plate, however, is observed to be maximum at fiber angle $\beta=90^{\circ}$. These results should be a valuable alternative for validating new computational techniques in future, due to the accuracy, simplicity and versatility of the present analysis. 


\section{References}

Leissa A.W., 1969. Vibration of Plates. NASA SP-169, Office of Technology Utilization, NASA, Washington, DC.

Rao, S. S., and Prasad, A. S., 1975. Vibrations of annular plates including the effects of rotatory inertia and transverse shear deformation. Journal of Sound and Vibration, Vol. 42, No 3, pp. 305-324.

Irie, T., Yamada G., and Takagi, K., 1982. Natural frequencies of thick annular plates. Journal of Applied Mechanics, Vol. 49, No 3, pp.633-638.

Han, J. B., and Liew, K. M., 1999. Axisymmetric free vibration of thick annular plates. International Journal of Mechanical Sciences, Vol. 41, No 9, pp. 1089-1109.

Liew, K. M., Xiang Y. and Kitipornchai, S. 1995. Research on thick plate vibration: a literature survey. Journal of Sound and Vibration, Vol. 180, No 1, pp. 163-176.

Lin, C. C., and Tseng, C. S. 1998. Free vibration of polar orthotropic laminated circular and annular plates. Journal of Sound and Vibration, Vol. 209, No 5, pp. 797-810.

Kang, J. H., and Leissa, A. W., 1998. Three-dimensional vibrations of thick, linearly tapered, annular plates. Journal of Sound and Vibration, Vol. 217, No 5, pp. 927-944.

So, J., and Leissa, A. W., 1998. Three-dimensional vibrations of thick circular and annular plates. Journal of Sound and Vibration, Vol. 209, No 1, pp. 15-41.

Liew, K. M., and Yang, B., 1999. Three-dimensional elasticity solutions for free vibrations of circular plates: a polynomials-Ritz analysis. Computer methods in applied mechanics and engineering, Vol. 175, No 1, pp. 189-201.

Liew, K. M., and Yang, B., 2000. Elasticity solutions for free vibrations of annular plates from three-dimensional analysis. International Journal of Solids and Structures, Vol. 37, No 52, pp. 7689-7702.

Liew, K. M., and Liu, F. L., 2000. Differential quadrature method for vibration analysis of shear deformable annular sector plates. Journal of Sound and vibration, Vol. 230, No 2, pp. 335-356.

Wu, T. Y., Wang, Y. Y., and Liu, G. R., 2002. Free vibration analysis of circular plates using generalized differential quadrature rule. Computer Methods in Applied Mechanics and Engineering, Vol. 191, No 46, pp. 5365-5380.

Zhou, D., Au, F. T. K., Cheung, Y. K., and Lo, S. H., 2003. Three-dimensional vibration analysis of circular and annular plates via the Chebyshev-Ritz method. International Journal of Solids and Structures, Vol. 40, No 12, pp. 3089-3105.

Wang, X., and Wang, Y., 2004. Free vibration analyses of thin sector plates by the new version of differential quadrature method. Computer Methods in Applied Mechanics and Engineering, Vol. 193, No 36, pp. 3957-3971.

Hosseini-Hashemi, S., Es'Haghi, M., Taher, H. R. D., and Fadaie, M., 2010. Exact closed-form frequency equations for thick circular plates using a third-order shear deformation theory, Journal of Sound and Vibration, Vol. 329, pp. 3382-3396.

Hosseini-Hashemi, S., Rezaee, V., Atashipour, S. R., and Girhammar, U. A., 2012. Accurate free vibration analysis of thick laminated circular plates with attached rigid core, Journal of Sound and Vibration, Vol. 331, pp. 5581-5596.

Senjanovic, I., Hadzic, N., Vladimir, N., and Cho, D. S., 2014. Natural vibrations of thick circular plate based on the modified Mindlin theory, Archives of Mechanics, Vol. 66(6) , pp. 389-409.

Sharma, A., 2014. Free vibration of moderately thick antisymmetric laminated annular sector plates with elastic edge constraints, International Journal of Mechanical Sciences, Vol. 83, pp.124-132.

Powmya, A., and Narasimhan, M. C. 2015. Free vibration analysis of axisymmetric laminated composite circular and annular plates using Chebyshev collocation, International Journal of Advanced Structural Engineering, pp. 1-13.

Kang, J. H., 2003. Three-dimensional vibration analysis of thick, circular and annular plates with nonlinear thickness variation. Computers \& structures, Vol. 81, No 16, pp. 1663-1675.

Liu, C. F., and Lee, Y. T., 2000. Finite element analysis of three-dimensional vibrations of thick circular and annular plates, Journal of Sound and Vibration, Vol. 233, No 1, pp. 63-80.

Malekzadeh, P., Shahpari, S. A., and Ziaee, H. R., 2010. Three-dimensional free vibration of thick functionally graded annular plates in thermal environment. Journal of Sound and Vibration, Vol. 329, No 4, pp. 425-442.

Liew, K. M., Han, J. B., Xiao, Z. M., and Du, H., 1996. Differential quadrature method for Mindlin plates on Winkler foundations. International Journal of Mechanical Sciences, Vol. 38, No 4, pp. 405-421.

Zhou, D., Lo, S. H., Au, F. T. K., and Cheung, Y. K., 2006.Three-dimensional free vibration of thick circular plates on Pasternak foundation. Journal of Sound and Vibration, Vol. 292, No 3, pp. 726-741.

Hashemi, S. H., Taher, H. R. D., and Omidi, M., 2008. 3-D free vibration analysis of annular plates on Pasternak elastic foundation via p-Ritz method. Journal of Sound and Vibration, Vol. 311, No 3, pp. 1114-1140.

Houmat, A., 2004. Three-dimensional hierarchical finite element free vibration analysis of annular sector plates. Journal of sound and vibration, Vol. 276, No 1, pp. 181-193.

Komur, M. A., Sen, F., Ataş, A. , and Arslan, N. , 2010. Buckling analysis of laminated composite plates with an elliptical/circular cutout using FEM, Advances in Engineering Software, Vol. 41, pp. 161-164.

Chen, D. Y., and Ren, B. S., 1998. Finite element analysis of the lateral vibration of thin annular and circular plates with variable thickness". Journal of Vibration and Acoustics, Vol. 120, pp. 747-752. 
Liang, B., Zhang, S. F., and Chen, D. Y., 2007 . Natural frequencies of circular annular plates with variable thickness by a new method, International journal of pressure vessels and piping, Vol. 84, pp. 293-297.

Ranjan, V., and Ghosh, M.K., 2009 . Transverse vibration of spinning disk with attached distributed patch and discrete point masses using finite element analysis, International Journal of Engineering, Science and Technology, Vol. 1, No. 1, 2009, pp. 7489.

Malekzadeh, P., Afsari, A., Zahedinejad, P., and Bahadori, R., 2010. Three-dimensional layerwise-finite element free vibration analysis of thick laminated annular plates on elastic foundation, Applied Mathematical Modelling, Vol. 34, No 3, pp. 776-790.

Reddy J.N., 2004. Mechanics of Laminated Composite Plates and Shells: Theory and Analysis. CRC Press, Boca Raton, FL, Second Edition.

ANSYS Inc., 2009. ANSYS 12.0 reference manual.

\section{Biographical notes}

Dr N. D. Mittal is a faculty member in the department of Mechanical Engineering, Maulana Azad National Institute of Technology, Bhopal

Sumit Khare is research student in the department of Mechanical Engineering, Maulana Azad National Institute of Technology, Bhopal.

Received November 2015

Accepted March 2016

Final acceptance in revised form June 2016 